\title{
Medições de radiações ionizantes e chuvas na região tropical do Brasil - dinâmica nos tempos
}

\section{lonizing radiation and rainfall measurements in the Brazilian tropical region - dynamics in the times}

Inacio Malmonge Martin ${ }^{1}$, Rodrigo Rezende Fernandes de Carvalho ${ }^{1}$, Rafael Augusto Gomes ${ }^{1}$, Marcelo Pego Gomes ${ }^{1}$

\section{RESUMO}

No período entre 07 de março e 28 de junho de 2017, foram monitorados os raios-gama de baixa energia e as chuvas a cada minuto em São José dos Campos, São Paulo, Brasil. Nesse período, foi possível observar o processo dinâmico que ocorre entre a presença de radiação ionizante de baixa energia e variação da intensidade da chuva em $\mathrm{mm} / \mathrm{min}$ na região. Esta correlação positiva de chuva/radiação é muito perceptível na região tropical do Brasil devido à presença da desintegração do urânio ${ }^{238} \mathrm{U}$ para rádio ${ }^{226} \mathrm{Ra}$, chegando ao radônio ${ }^{222} \mathrm{Rn}$ com emissão de partículas $\alpha$ e radiação gama de baixa energia. $A$ chuva interfere na presença da exalação local do gás radônio causando a lavagem deste gás na baixa atmosfera, aumentando a intensidade de radiação medida momentaneamente no local. Este trabalho mostra esse processo observado no período mencionado, durante o qual ocorreram climas chuvoso e seco.

Palavras-chave: Raios gama, Radiação ionizante, Análise de chuva.

\begin{abstract}
Between March 7 and June 28 of 2017, the low energy gamma rays and the rains were monitored every minute in São José dos Campos, São Paulo, Brazil. In this period, it was possible to see the dynamic process that occurs between the presence of low energy ionizing radiation and rain intensity variation in $\mathrm{mm} / \mathrm{min}$ in the region. This positive correlation of rain/radiation is very noticeable in the tropical region of Brazil due to the decay of ${ }^{238} \mathrm{U}$ uranium to radium ${ }^{226} \mathrm{Ra}$, reaching radon ${ }^{222} \mathrm{Rn}$ with emission of particles $\alpha$ and low energy gamma radiation. The rain interferes with the presence of local exhalation of the radon gas, causing its washing in the low atmosphere, increasing the intensity of radiation measured momentarily in the place. This work shows this process measured during the period mentioned, in which occurred both rainy and dry climates.
\end{abstract}

Keywords: Gamma-ray, lonizing radiation, Rainfall analysis.

1'Departamaneto de Ciência e Tecnologia Aeroespacial - Instituto Tecnológico de Aeronáutica- Departamento de Física - São José dos Campos/SP - Brasil. Autor correspondente: Inacio Malmonge Martin | Departamaneto de Ciência e Tecnologia Aeroespacial - Instituto Tecnológico de AeronáuticaDepartamento de Física - | Praça Marechal Eduardo Gomes, 50 | CEP 12.228-900 - São José dos Campos/SP - Brasil | inaciomalmonge@uol.com.br Recebido: 23 Jan. 2018 | Aceito: 23 Mar. 2018 


\section{INTRODUÇÃO}

$\mathrm{Na}$ interface solo/ar da superfície da Terra, a radiação ionizante é composta principalmente de gás radônio, da radiação telúrica do solo e da radiação dos raios cósmicos primário e secundário. No entanto, é difícil separar ao longo do tempo a intensidade da radiação ionizante que emana de cada componente à medida que as energias se sobrepõem. A radiação telúrica está dada por ${ }^{238} \mathrm{U},{ }^{235} \mathrm{U},{ }^{40} \mathrm{~K},{ }^{232} \mathrm{Th}$ e é constante para cada região ${ }^{1}$. O gás radônio que vem da desintegração de ${ }^{238} \mathrm{U}$ da crosta terrestre ${ }^{2}$ para Ra-226 e Rn-222, chega nos isótopos ${ }^{214} \mathrm{~Pb},{ }^{214} \mathrm{Po}$ e ${ }^{214} \mathrm{Bi}$, gerando radiação a e gama. A radiação cósmica primária consiste principalmente de prótons galácticos e extragalácticos e do Sol, com energia muito alta que interage com a atmosfera da Terra produzindo o EAS (Extensive Air Showers) ${ }^{3}$. A eficiência dessa interação é máxima quando ocorre em altitudes entre 15 e $17 \mathrm{~km}$ nos trópicos, que formam raios cósmicos secundários com componentes muonicas, mesônicas e neutronicas que chegam à superfície da Terra na região ${ }^{4}$. Essas radiações causam problemas de saúde para a tripulação e os passageiros da aviação civil e estão presentes no início da estratosfera chamada máximo de Pfotzer. No entanto, essa componente contribui menos para a concentração de radiação na superfície da Terra. Outra possível fonte de radiação ionizante na atmosfera inferior da Terra é produzida por descargas elétricas entre nuvens-terra, terra-nuvens e nuvens-nuvens. Raios- $\mathrm{X}$, raios gama, nêutrons e partículas beta são formados pelo cone do relâmpago ${ }^{5}$. Outras fontes de radiação ionizante são as produzidas em clínicas médicas, dentais e hospitais, mas estas são principalmente controladas em pequenas áreas. $\mathrm{O}$ objetivo deste trabalho foi monitorar os raios-gama de baixa energia e as chuvas a cada minuto em São José dos Campos, São Paulo, Brasil.

\section{METODOLOGIA}

O detector de raios gama para o intervalo de energia de $200 \mathrm{keV}$ a 10,0 MeV consiste em um cristal cintilador de iodeto de sódio de 3 polegadas de altura por 3 polegadas de diâmetro dopado com tálio. Esse cristal é diretamente acoplado a uma fotomultiplicadora (PM) que registra os pulsos vindos do cintilador com amplificação e um conversor analógico digital, com sinais registrado por um computador ${ }^{6}$. Esse conjunto experimental (Fig. 1) localiza-se na sala interna de uma torre de $25 \mathrm{~m}$ de altura em relação ao solo (Torre ACA).

$\mathrm{O}$ cintilador acoplado à fotomultiplicadora está envolvido numa camada fina de alumínio para torná-lo portátil. O conjunto (cintilador + eletrônica associada + aquisição de dados) depende apenas de um laptop com bateria carregada para medir a radiação durante até 5 horas contínuas. No entanto, para séries de medidas longas, usa-se rede elétrica ou energia fotovoltaica. O cintilador e eletrônica associada foram calibrados em termos de energia e intensidade de contagens por minuto no laboratório de física experimental de ensino do ITA utilizando fontes

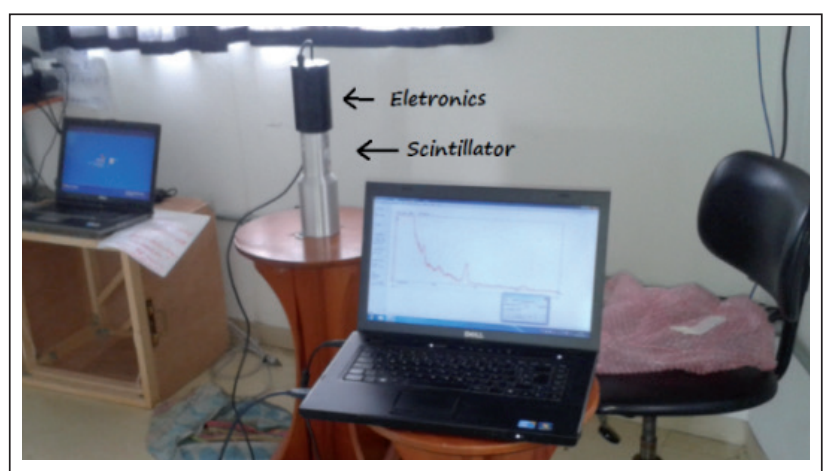

Figura 1: Vista do cintilador gama com eletrônica associada e computador.

radioativas e um analisador espectral de contagens $\times$ energia no intervalo de 0,2 a $10 \mathrm{MeV}$ (Milhão de eléctron Volt) ${ }^{7,8}$.

\section{RESULTADOS E DISCUSSÃO}

As medidas foram realizadas entre os dias 07 de março e 28 de junho de 2017 no equipamento mostrado na Fig. 1, na Torre ACA, com $25 \mathrm{~m}^{\text {de altura }}{ }^{7}$. Cada medida foi feita em intervalos de 1 minuto. Assim, foi possível verificar tempos com chuvas e a dinâmica da radiação ionizante na região. A Fig. 2 mostra, durante todo o período estudado, 12 chuvas, vistas através dos picos de aumento da radiação provocado por elas.

Na Fig. 2 observa-se chuva moderada, entre 43 e 43,5 $\times 10^{3}$ min a partir do início do monitoramento da série de radiação. Ampliando-se o gráfico nessa região vemos, na Fig. 3, o detalhe da ocorrência da chuva via medida da radiação ionizante (raios gama) nesse intervalo. Esses detalhes são importantes para melhor definir os efeitos da radiação provocada pelas chuvas.

A Fig. 4 apresenta uma amplificação próxima ao intervalo de 72000 min após o início das medidas. Esse intervalo representa a passagem de uma frente fria que registrou, durante cinco dias, um pequeno aumento da radiação gama local com chuva intensa na região ao final desse período. Essa dinâmica meteorológica é bastante observada na região, provocando chuvas e coberturas de nuvens menos ou mais intensas.

Durante o período de 129,5 a $130,5 \times 10^{3}$ min houve chuvas intermitentes com intensidades variadas (Fig. 5).

Outra chuva moderada que alterou a intensidade de radiação gama medida pode ser observada no tempo de $\sim 141 \times 10^{3} \mathrm{~min}$ após início do monitoramento, como mostra a Fig. 6. No dia anterior à chuva a temperatura local subiu até $30^{\circ} \mathrm{C}$ devido à chegada de uma frente fria vinda do sul do Brasil.

Analisando a dinâmica da radiação gama medida de minuto a minuto em função do tempo em local fixo pode-se observar também a dinâmica da variação das chuvas ocorridas no mesmo local. Na Fig. 7 mostra-se o espectro das chuvas em função do tempo, medidas no mesmo período e no mesmo local sempre com intervalo de um minuto entre cada medição. 


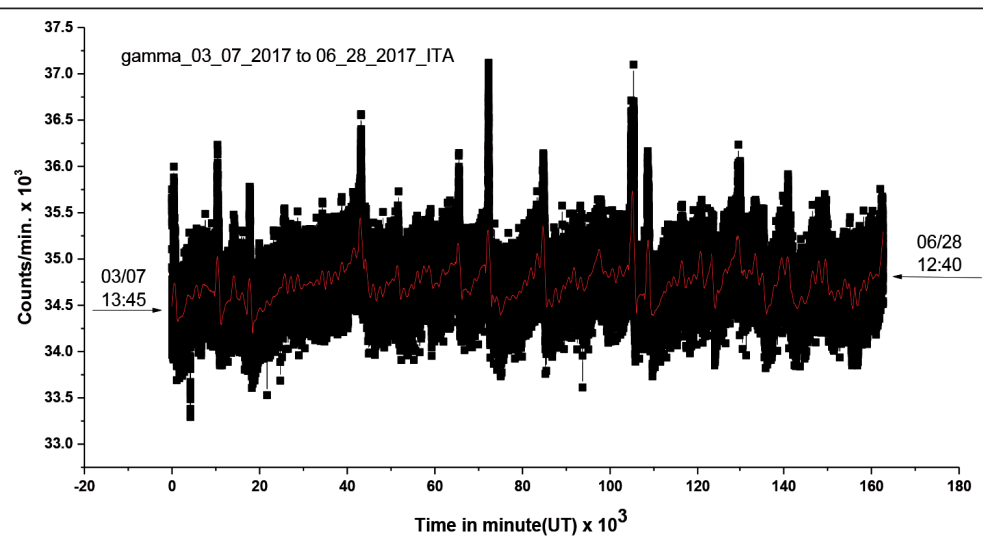

Figura 2: Medidas da radiação gama no intervalo de energia de 0,2 a 10,0 MeV a cada minuto entre 07 de março e 28 de junho de 2017.

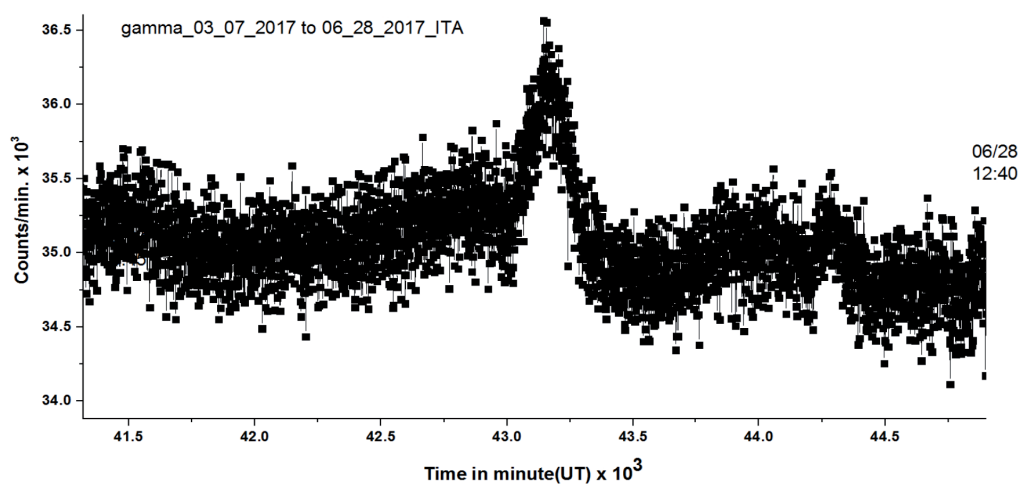

Figura 3: Monitoramento de chuva através da radiação gama no intervalo entre 43 e 43,5 × 10³ min após início das medidas.

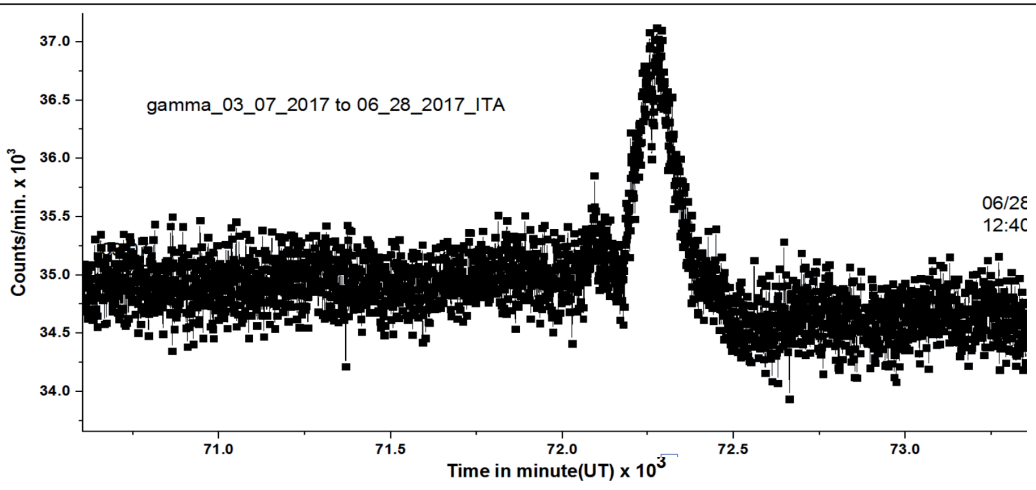

Figura 4: Intervalo do monitoramento da radiação entre 72,0 e $72,5 \times 10^{3} \mathrm{~min}$.

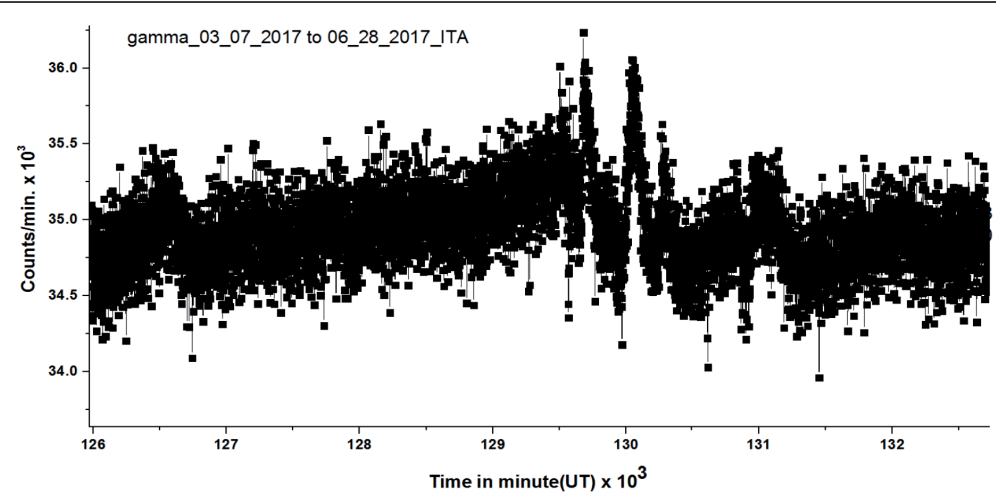

Figura 5: Chuvas intermitentes com intensidades variadas, entre 129,5 e 130,5 × $10^{3} \mathrm{~min}$. 


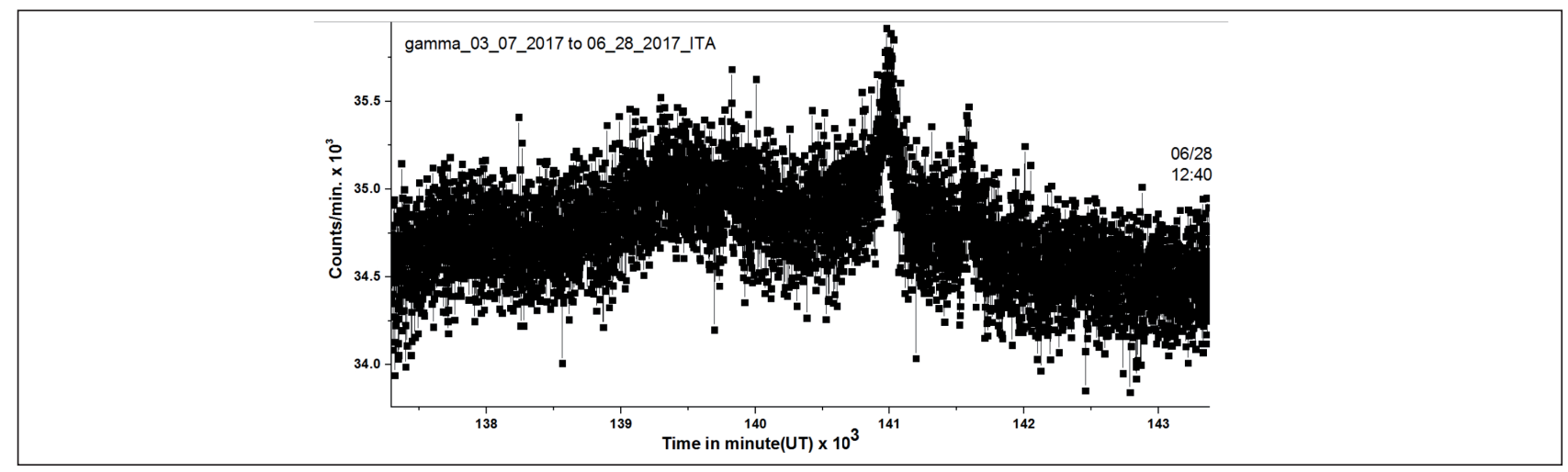

Figura 6: Chuva moderada verificada no tempo de $~ 141000 \mathrm{~min}$.

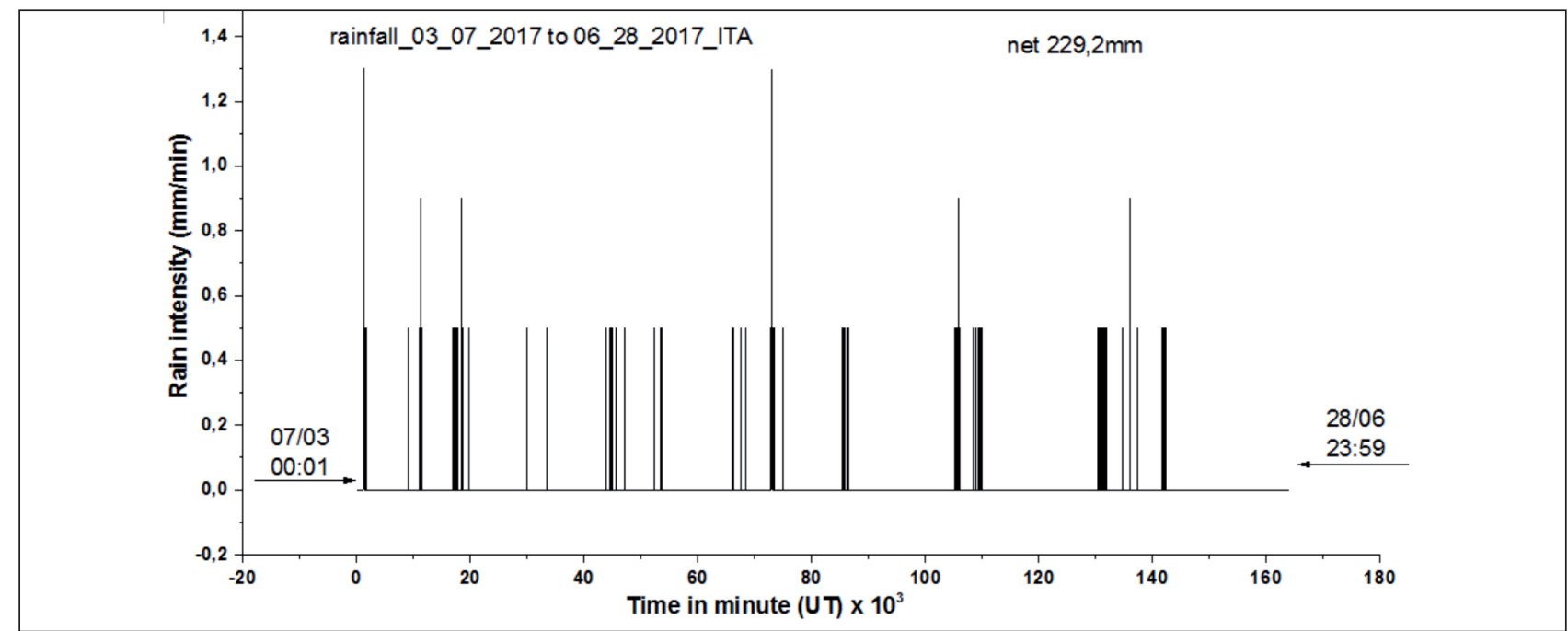

Figura 7: Espectro em função do tempo das chuvas ocorridas a cada minuto entre 07 de março e 28 de junho de 2017.

Nota-se, na Fig. 7, que em torno de $43 \times 10^{3}$ min ocorre uma chuva moderada, observada na Fig. 3 da radiação. Entre 72 e $73 \times 10^{3} \mathrm{~min}$ acontece uma chuva intensa, correspondente ao aumento intenso da radiação, verificado na Fig. 4. Cuidadosamente pode-se correlacionar a Fig. 7 (intensidade de chuvas no período) com a Fig. 2 (intensidade de radiação gama no período). Logo, na região tropical do Brasil, devido à exalação do gás radônio, torna-se possível correlacionar intensidade de radiação ionizante com intensidade de chuvas ocorridas no mesmo local.

\section{CONCLUSÃO}

Neste trabalho, utilizando-se um detector de raios gama no intervalo de energia 0,2 a $10,0 \mathrm{MeV}$, foi possível correlacionar medidas de radiação com medidas de chuvas da região efetuadas no período 07 de março a 28 de junho de 2017. Essa correlação positiva chuva/radiação é perceptível na região tropical do Brasil devido à presença do decaimento do urânio ${ }^{238} \mathrm{U}$ no radio ${ }^{226} \mathrm{Ra}$, e deste no radônio ${ }^{222} \mathrm{Rn}$, com emissões de partículas a e radiação gama de baixa energia. Uma vez efetuada a calibração entre a intensidade de água e a intensidade de radiação gama no local, é possível medir intensidade de chuvas através do monitoramento da radiação na região. No momento, outro trabalho está em andamento, com o intuito de mostrar essa calibração com testes efetuados no laboratório do ITA ${ }^{8}$.

\section{REFERENCES}

1. Bui-Van NA, Martin IM, Turtelli Jr A. Measurements of natural radioactivity from natural, industrial and atmospheric depths. Ciência e Cultura. Suplemento. 1998;40(7):407.

2. Fujinami N. Study of radon progeny distribution and radiation dose rate in the atmosphere. Japanese J Health Phys. 2009;44(1):8994. Disponível em: https://doi.org/10.5453/jhps.44.89

3. Grieder PKF. Extensive air showers: high energy phenomena and astrophysical aspects, Springer: Verlag Berlin Heidelberg; 2010.

4. Malyshevsky VS, Fomin GV. Electromagnetic radiation in the atmosphere generated by excess negative charge in a nuclearelectromagnetic cascade. Russ Phys J. 2017;59(9):1367-1372 Disponível em: https://doi.org/10.1007/s11182-017-0918-9 
5. Jayanthi UB, Gusev AA, Neri JACF, Villela T, Pinto Jr O, Pugacheva Gl, et al. Ground gama radiation associated with light and rain precipitation. In: Acharya S, Gupta S, Jagadeesan P, Jain A, Karthikeyan S, Morris S, et al. editors. Proceedings of the 29th International Cosmic Ray Conference; 2005 Aug 3-10; Pune (India). 2005;1:177-180.

6. Ravisankar R, Vanasundari K, Chandrasekaran A, Rajalakshmi A, Suganya M, Vijayagopal P, et al., Measurement of natural radioactivity in building materials of namakkal, tamil nadu, india using gamma-ray spectrometry. Applied Radiation and
Isotopes. 2012;70(4):699-704. Disponível em: https://doi. org/10.1016/j.apradiso.2011.12.001

7. Boardman B J. Wilmington: Aware Electronic Corp. [Acesso em 2017 Jul. 3]. Disponível em: www.aw-el.com

8. Silva MC, Vilela DC, Migoto VG, Gomes MP, Martin IM, Germano JSE, Ionizing Radiation Measurements using low cost Instruments for teaching in college or high-school in Brazil. Phys Educ. 2017;52(6):064004. Disponível em: https:// doi.org/10.1088/1361-6552/aa8420 